\title{
Enhancing the Role of Machine Learning in STEM Disciplines through Supervised Undergraduate Research Experiences
}

\author{
Yaser M. Banadaki \\ Department of Computer Science, \\ Southern University, Baton Rouge, USA
}

\begin{abstract}
Machine learning (ML), as a branch of Artificial Intelligence (AI), is a field of computer science that has seen rapid development over the past few years. ML promises to make computers capable of discovering meaningful information and making predictions about the outcome. Despite the significance of this field and high demand, knowledge of how to effectively provide ML-based research experience to STEM students is scarce. This paper focuses on the role of ML education in non-CS majors to improve the students' computing and analytical skills across a range of domain-specific problems. The paper studies four examples of ML-intensive STEM projects for supervised undergraduate research experiences (SURE) in Mechanical Engineering, Biology, Physics, and Cybersecurity. The developed projects include the applications of $M L$ for improving additive manufacturing, automating microscopy images analysis, identifying the quantum optical modes, and detecting the internet of things (IoT) network intrusion. The paper aims to provide some guidelines to effectively educate the next generation of STEM undergraduate and graduate students and prepare STEM professionals with interdisciplinary knowledge, skills, and competencies in ML and data science. The students understand how to solve big data problems effectively in their disciplines and confront the new challenges in a computationally-driven world.
\end{abstract}

\section{Introduction}

All STEM students must have access to highquality education to ensure our nation's economic growth [1]. STEM occupations account for more than $50 \%$ of the employment in major industries, which outpaces the production of STEM degrees in America [2]. It is now abundantly clear that the economic health of the nation depends on a robust infrastructure based on STEM foundations. Yet, we have a vast talent pool comprising underrepresented student groups (minority, female, and low-income) that, unfortunately, remain under-utilized because of the lack of opportunity to have access to high-quality education. The McKinsey Report [3] estimates a shortage of almost 200,000 people with deep analytical skills, and 1.5 million managers and analysts to analyze big data and make decisions based on their findings in the United States. Similarly, it has been predicted [4] that in many states, mathematical and computing occupations will display the largest ten-year growth of any sector by 2022 .

Furthermore, the National Center for Women \& Information Technology [5] projected that up to 77\% of future job openings could be filled by people with computing degrees. Despite the job opportunities, states with large minority populations like Louisiana had only 365 ( $18 \%$ female) and 455 (24\% female) computer science (CS) graduates in 2015 and 2017, respectively [6]. Although the trend is positive, these numbers are far from the current 2,333 computing job openings in this state [6]. African Americans fill 5.5\% of STEM jobs at levels disproportionate to their overall representation (11\%) in the workforce [7]. On top of the small number of CS majors, most science and engineering graduates, especially among underrepresented minorities, do not acquire the essential skills and dispositions needed to succeed in a computationally-driven world.

Artificial Intelligence (AI) and Machine Learning (ML) makeup not only the largest occupation groups in STEM [2] but also enable underserved students to develop and apply the knowledge and skills in STEM disciplines. $\mathrm{AI}$ and $\mathrm{ML}$ are often taught in a decontextualized manner in which these topics are presented in a CS class that is separated from their application in other content areas. This approach is inefficient in that students do not see the connection and usefulness of this field across their many applications in other disciplines. As such, the CS students do not perceive the significance of $\mathrm{AI}$ and ML as creative and applicable topics that can help solve problems in various contexts. Judd et al. [8] showed that integrating advanced computing subjects in non-CS curricula promotes students' interest and inspires them to use the power of computing to solve real-world domain-specific problems. Similarly, the non-CS students do not have the opportunity to engage in the ML-based research for their capstone 
projects. The number of courses offering in the areas of computational science in STEM disciplines is minimal, and mostly there is no AI, ML, or data science courses offering in non-CS STEM programs. Also, enrollment in standard CS course sequences does not always serve these diverse STEM student populations well, so that a few non-CS students enroll in advanced ML courses. The average number of students enrolling in technical CS courses from other STEM majors is limited, and most of the mandatory courses in STEM majors have no modules for data science and analysis techniques.

Of equal urgency is the need to improve the role of machine learning in the undergraduate STEM capstone projects in addition to improving and introducing comprehensive computing and data curricula in these majors. Acquiring computing skills is essential for innovation and competitiveness that enables many underserved students to navigate successful STEM career pathways. Recent studies [9, 10] found that students' exposure to advanced CS techniques, such as ML as new research tools for a variety of majors, can potentially engage them in computing and place them on a trajectory for future CS-related STEM education and employment. To maximize the impact of computing, AI, ML, and data science in other disciplines, the universities need not only carefully plan how to align CS courses for different STEM fields but also encourage computingenhanced experiences for STEM capstone projects. The Next Generation Science Standards (NGSS) explicitly calls out CS topics in their practices as a tool to effectively engage students in CS experience [11]. Integrating ML and STEM not only advances the students' knowledge and skills in CS but also promotes deeper learning of STEM concepts. Applying ML techniques in STEM capstone projects improves how and what students learn in the classroom by supporting four fundamental characteristics of learning: active engagement, participation in groups, frequent interaction and feedback, and connections to real-world contexts.

The goal of this paper is to encourage the implementation of interdisciplinary ML-intensive projects for STEM students. The rest of the article is organized as follows. Section 2 presents the guideline to implement an interdisciplinary ML-intensive STEM supervised undergraduate research experience. Section 3 provides examples of four interdisciplinary projects to highlight the critical role of ML literacy across all non-CS STEM disciplines and encourage faculty and students in the adoption of ML techniques for real-world problem-solving. The four MLintensive STEM projects include the applications of ML for improving additive manufacturing, automating microscopy images analysis, identifying the quantum optical modes, and detecting IoT network intrusion. The last section draws summarizing conclusions and future work.

\section{Machine learning in STEM SURE}

ML, as a branch of AI, is increasingly central to innovation across a wide range of disciplinary domains. Thus, it is crucial to encourage STEM students to explore a connection between their domain-specific capstone projects and the interdisciplinary potentials of the ML field. To support effective ML-enhanced domain-specific capstone projects, supervised undergraduate research experiences (SURE) can be designed. The SURE provides the opportunity for STEM students to have both the CS and non-CS faculty advisors. The interdisciplinary research subjects bridge CS and STEM domains to increases the research experience and participation in computational and data science and motivate students to pursue graduate degrees or careers in CS-intensive STEM fields. Under the umbrella of machine learning, SURE transfers the contents of computer science, computational science, and data analytics to STEM students. The SURE research can be part of their senior capstone project or thesis required for graduation supervised by both CS and non-CS faculty. To implement an effective SURE research, the CS mentors need first to learn the students' computing skills to develop an effective interdisciplinary ML research project and modify/adjust the computational tasks accordingly. Mentoring relationships need to consider students' personal goals, needs, previous educational experiences, and learning styles to effectively promote STEM students' interests in ML research and education. The SURE's interdisciplinary projects will establish foundations for a strong, trusting, and supportive relationship between students and mentors that flows both directions instead of only from mentors down to students. As such, the faculty mentors could effectively stress the importance of CS skills for developing their theoretical and experimental concepts in their disciplines. The common goal of the CS faculty mentor is to support computing literacy, computational thinking, data analysis, and programming skills to advance the adoption of ML techniques for solving and modeling real-world problems in STEM disciplines. As students become more proficient with the fundamentals of ML applications and data science, the faculty mentors need to increase attention to their progress both as researchers by acting as a consultant, and as professionals by suggesting lines of inquiry and options for solving domain-specific problems.

The ML-intensive SURE project harnesses the role of future ML developments in STEM disciplines. It empowers undergraduate students to confront the challenges in computational and data-enabled sciences to analyze and make decisions based on their findings. The SURE projects focusing on ML application could provide a forum through which undergraduate students acquire the confidence and 
scientific preparation required to enroll in a graduate program and pursue a professional career related to machine learning. SURE's ML-intensive interdisciplinary projects could improve a shortage of workforce with deep analytical skills in the United States. Even though the students could learn how to use computational tools for their domain-specific applications through attending CS-related classes, many technical details remain a black box for many students. For instance, choosing the optimal hyperparameters for neural networks is critical to have high performance and precision. However, it requires research experience to apply extensive trial and error to find the optimal values for the ML model. Without having enough skills, it becomes a huge problem for the students starting in the field. Enhancing the interdisciplinary ML-based projects, such as four examples of SURE projects designed in this paper, increase the number of qualified graduates with deep analytical skills for the nation's workforce needs.

In addition to individual mentors-mentees relationships, students will attend ML-related technical meetings and conferences. The program exposes students to additional areas of study and provides a guide for the development of their communication and presentation skills. Students have the opportunity to write ML-related domain-specific papers and presenting them to a large audience at conferences. It boosts undergraduate students' confidence and creates a sense of ownership. The program makes students interested in pursuing a graduate degree in their discipline with intensive computational and data sciences. It ignites a long-term interest in students to pursue the ML-knowledgeable STEM workforce. For STEM students, CS conferences will improve their understanding of the significance of employing computational and AI techniques to solve real big data problems. The students are encouraged and supported to participate in regional and national conferences, seminars, and workshops, where they present posters or papers and expose to the research in AI, ML, computational, and data science. The students have the opportunity to network with scholars who are leaders in the field, which positively impact on their career development. The CS conferences also update undergraduate students on the latest employment trends and internship opportunities in AI, ML, computational, and data science. Also, attending CS conferences provides a good knowledge of professional standards, ethical issues, job environment responsibility, balancing career, and personal life. The STEM SURE's goal is to provide a forum through which undergraduate students acquire the confidence and scientific preparation required to enroll in a graduate program and pursue a professional career related to AI, ML, computing, and data sciences. STEM SURE guides students toward becoming independent creators of knowledge or users of ML concepts to apply them to their disciplines, prepared for the career paths of their choice, and ready to move on to the next phase of professional life.

The impact of Interdisciplinary ML-based SURE projects on economic development is through producing qualified STEM graduates with the ability to collaboratively apply ML skills in their domainspecific problems and across a range of contexts and challenging issues in STEM. Improving the quality and number of underrepresented graduates will help fill the needs of more qualified STEM professionals in the nation.

\section{Examples of Interdisciplinary Machine Learning Projects for STEM SURE}

\subsection{CS + Mechanical Engineering SURE Project: Machine learning for improving additive manufacturing}

Additive manufacturing (AM) [12] or 3D printing is a technique of blending or depositing materials, layer upon layer, in precise geometric shapes. As AM is rapidly gaining viability in mass production, it is essential for students entering the workforce to understand how to use this technology. The parts built using the state-of-the-art powder-bed AM, however, have remarkable unpredictable mechanical properties. The attempts to improve the geometry and mechanical properties of final products in the $3 \mathrm{D}$ printing process were usually limited to the development of a typical control system using feedback from sensor measurement. Current AM systems only have limited sensing capability, and most of them are inaccessible to the users. Future AM needs to be a smart system that can perform self-monitoring, self-calibrating, and real-time quality self-controlling.

In this SURE research project, students learned how to employ the ML approach through a Deep Convolutional Neural Network (DCNN) [13] to detect the defects in printing the layers automatically. The students understood how to use a transfer learning approach based on Google's open-source Inceptionv3 model in the Tensorflow framework. Figure 1 shows the samples of designed and printed 3D objects that were collected for training the DCNN model. The developed DCNN model achieves a classification accuracy rate of $98.31 \%$, producing a precise feedback signal for a smart 3D printer to recognize any issues with the build itself and make proper adjustments and corrections without operator intervention. The ML model enhances the quality of the AM process, leading to manufacturing better parts with fewer quality hiccups, limiting waste of time and materials. Furthermore, this SURE project could train a new generation of engineers who bring ML-related skills to the AM workforce and any manufacturing business. The students who learn ML and apply it to AM can bring creativity to design and production. 


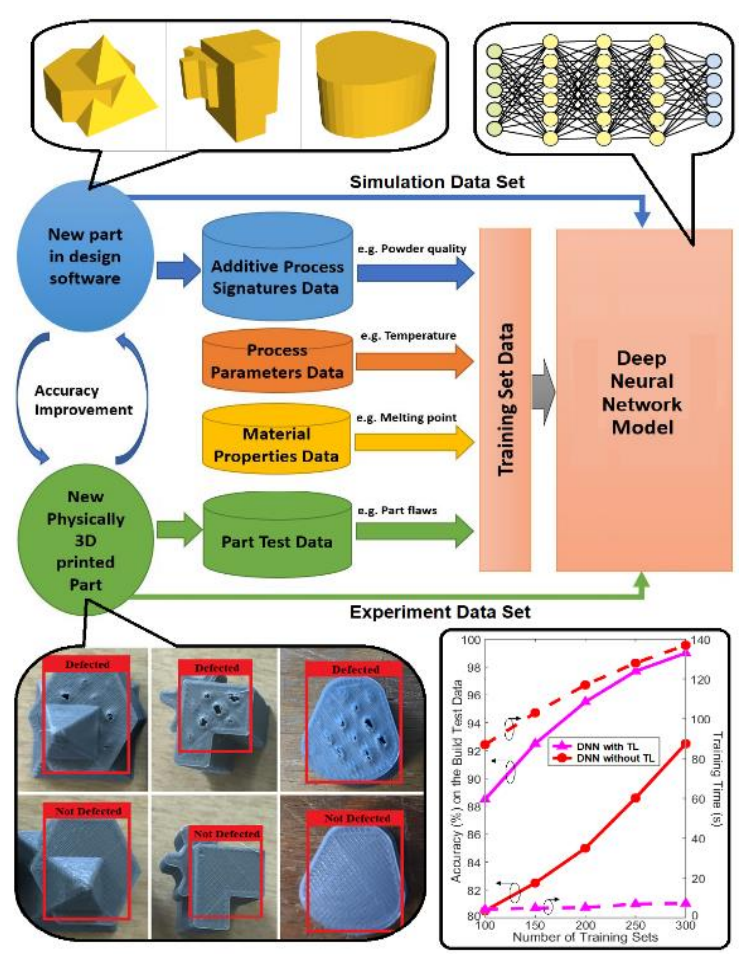

Figure 1. Smart 3D printers with higher performance were presented by training the deep neural network model using the transfer learning approach. The training datasets were collected from the additive process, materials, design software, builds inspections.

\subsection{CS + Biology SURE Project: Machine learning for automating microscopy images analysis}

The history of relations between biology and the field of machine learning is long because the artificial neural network was inspired from the organization of the visual nervous system. The general cell quantification tools are manual or semi-automated techniques that are time-intensive, cumbersome, and prone to human errors. Available analysis software is based on the assessment of fixed immunolabelled tissue samples, making it impossible to follow the dynamic development of neurite outgrowth. This SURE project develops an accurate and fully automated technique for the quantitative analysis of microscopy images. ML approach through a DCNN model has recently shown remarkable success in image-based data analysis resulting in a tremendous improvement in automated detection of complex morphologies [14]. This interdisciplinary SURE project integrates research and education in the areas of bio-imaging study and outreaches the state-of-theart $\mathrm{AI}$ research by training undergraduate students.

In this SURE project, the students learn how to employ an ML approach to generate an accessible bioimaging analysis tool for biologists to detect and quantitatively analyze cells in high-content microscopy images accurately. The experimental
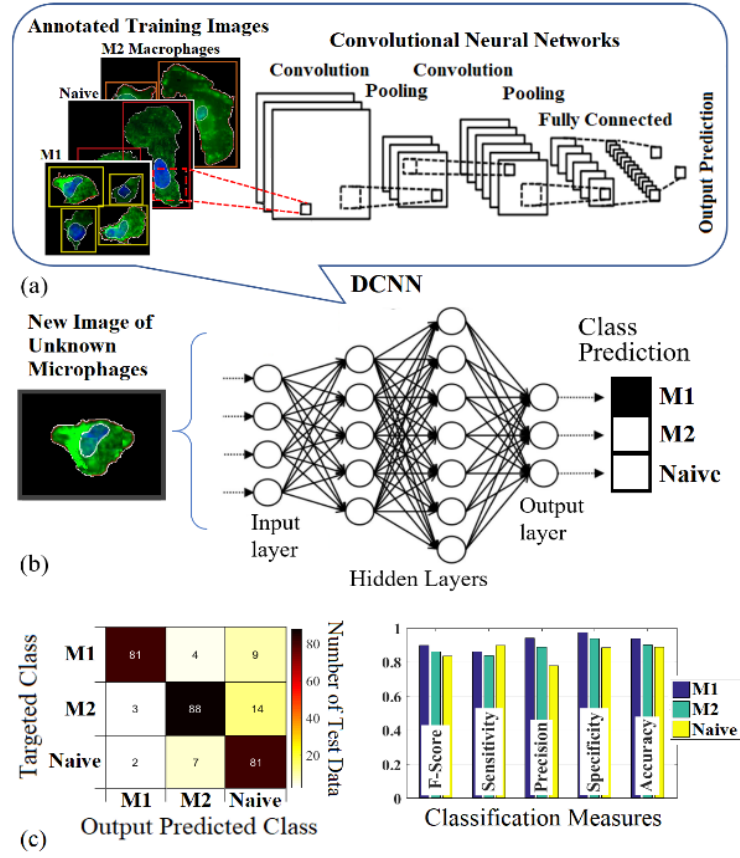

Figure 2: (a) Training procedure of DCNN using annotated cell images; (b) Schematics of DCNN that can automatically classify new unknown macrophages; and (c) Confusion matrix showing the detailed number of correctly classified and misclassified images of naïve, M1, and M2 macrophages, as well as five statistic classification measures.

dataset containing microscopy images are used to train and examine the performance of the DCNN approach. Two different training and validation data sets containing $80 \%$ and $20 \%$ images of annotated cell images are used to train and evaluate the DCNN model. The students learn how to train the DCNN model consists of an input and output layer together with multiple hidden layers convolutional, pooling, non-linear, or fully connected layers, among others. As shown in Fig.2, the DCNN provides automated, rapid, and accurate image analysis techniques to identify polarized macrophages M1 and M2 phenotypes and distinguish them from un-polarized macrophages and monocytes without the need for using multiple markers [15]. The automated model makes it possible to rapidly identify different macrophage phenotypes using cell morphology metrics as an indicator of their activation status. Given the complexity of M1/M2 identification in human macrophages, the approach is more straightforward, less resource-intensive, thus more widely adaptable for high throughput evaluation. Through this SURE project, students from biology learn how to deal with the creation and evaluation of algorithms that facilitate pattern recognition, classification, and prediction, based on models derived from existing data from microscopic images. Understanding machine learning methods enable biologist to improve the efficiency of discovery and interpretation of complex biological data. 


\subsection{CS + Physics SURE Project: Machine learning for identifying the quantum optical modes}

Physics is the foundational science, and optics is the science of the study of light in physics. Students with a background in optics technology use computational analysis and data science to design, model, and create optical sensing security products, light detection products, and products that use lasers. Laguerre-Gaussian (LG) optical modes are the solutions of the free-space Maxwell's equations in cylindrical coordinates [16]. LG modes have applications from optical communications to atomic physics. In the experimental laboratory, the CCD or CMOS cameras usually use to capture the mode information such as centroid and radius of a beam profile, but identifying the modes with human eyes is challenging, especially in higher modes. One good solution to automatically identify modes with high speed is to use ML methods.

The DCNN model is used to automatically classify the 16 different LG modes with multilayer convolution to extract features and combine the features automatically, as shown in figure 1. The students learned how to obtain the training and validation accuracy versus epoch (number of times the entire dataset is passed through the DCNN) for two training datasets of only simulated images and both simulated and experimental images. The DCNN model with theory dataset reaches the maximum accuracy of $100 \%$ after seven epochs while training with the second dataset that includes both theory and experiment images needs 33 epochs to reach the same maximum accuracy due to the diversity of the images. The validation accuracy of $100 \%$ is achieved after 58 and 350 epochs for the two training datasets, one consisting of only the simulated images and the other containing both the simulated and experimental images, respectively. Maximum accuracy of $92 \%$ and $81.7 \%$ are achieved after six epochs on the simulated dataset and both simulated and experimental datasets, respectively.

In this SURE project, students learn how to apply ML algorithms to reconstruct and detect the quantum properties of optical systems. The interdisciplinary field of combining machine learning and physics is growing rapidly, and exciting progress is being made. Through this project, students learn how the marriage of the ML and quantum physics may give birth to a new research frontier that could automate the laboratory procedure and facilitate the experimental techniques. The project motivates students to use machine learning to discover new fundamental concepts in physics and effectively enhance basic conceptual understanding of physics. This strategy paves the way to transform the educational aspects of our current laboratory experiments into new domains.

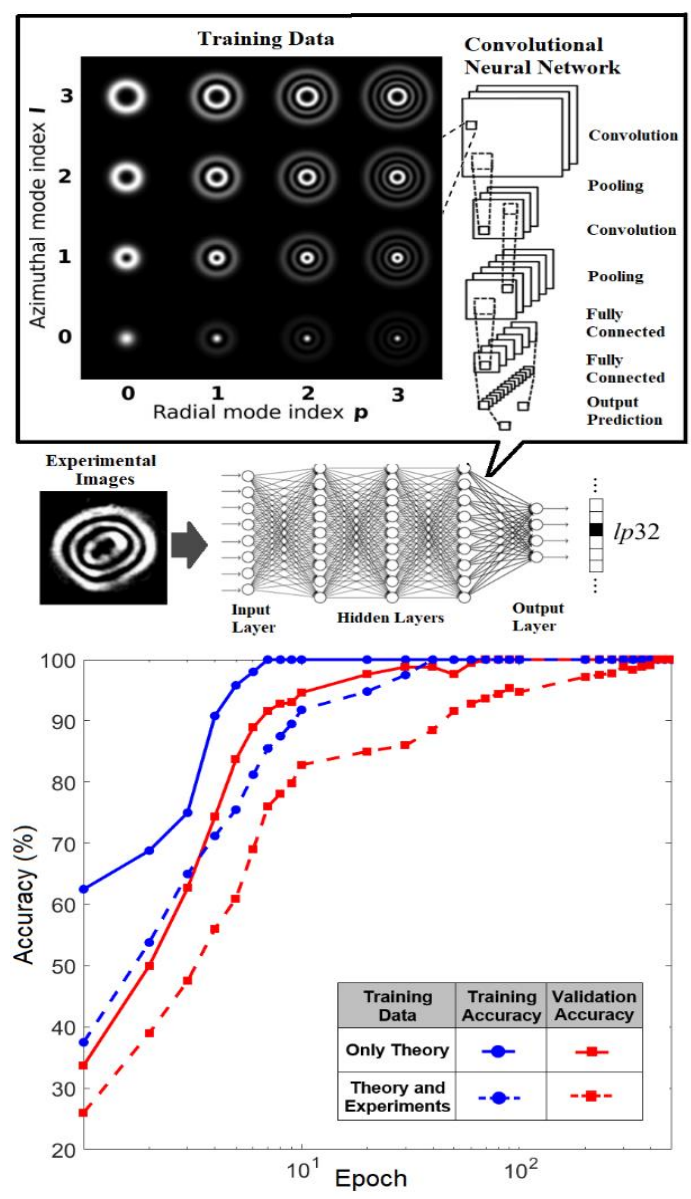

Figure 3. The training dataset of the first 16 LG modes is passed to the CNN model that extracts spatial features using aggregation layers that are eventually combined at the top layer to predict and classify the new image of LG mode. The graph shows the training and validation accuracies versus epoch for two CNN models that are trained using the datasets of only simulated images (solid lines) and both simulated and experimental images (dashed lines).

\subsection{CS + Cybersecurity SURE Project: Machine learning for detecting IoT network intrusion}

The internet is one of the most used inventions to date, and every day billions of people access the web to handle a wide variety of tasks such as email, banking, and store data. Internet of Things (IoT) has grown up rapidly that makes security and central privacy subjects for network design. The detection of anomaly attacks is the first and crucial point for avoiding any interruption in the connection. IoT includes wearable technology, kitchen robots, electric cars, mobile phones, etc. Despite network security policies and topics that should cover unknown third party attempts, Canedo et al. [17] explained that security is one of the weakest areas in IoT. Hasan et al. [18] studied how ML can detect anomaly attacks on IoT systems. Lawal et al. [19] propose different network anomaly reduction schemes in IoT networks. 
The available dataset of cybersecurity, CICIDS2017 [20] is used for training the neural network. The datasets contain a sufficient amount of benign data, the same types of attack data, and an equal amount of feature sets. Figure 4 (a) details the percentage break down of each type of attack data included in the dataset. Figure 4 (b) shows the accuracy of training and testing processes versus epoch for the learning rate of 0.1 and different values of batch sizes and the number of training data. It can be observed that the larger batch size refers to the number of training samples propagated through the network, which increases the test accuracy of the DCNN model to $80 \%$ for the model trained by 100 samples of training data. Increasing the size of the training data to 1000 improves the test accuracy of the model to $85 \%$.

In this SURE project, students learn how ML ensures the protection and monitoring of information and data in a network by detecting anomalies and intrusions in networks in real-time. The project helps students to understand how the marriage of two CS concepts, ML and cybersecurity, can create a smarter network by analyzing the traffic with better accuracy, increasing the correct alerts of bad and good network activities.
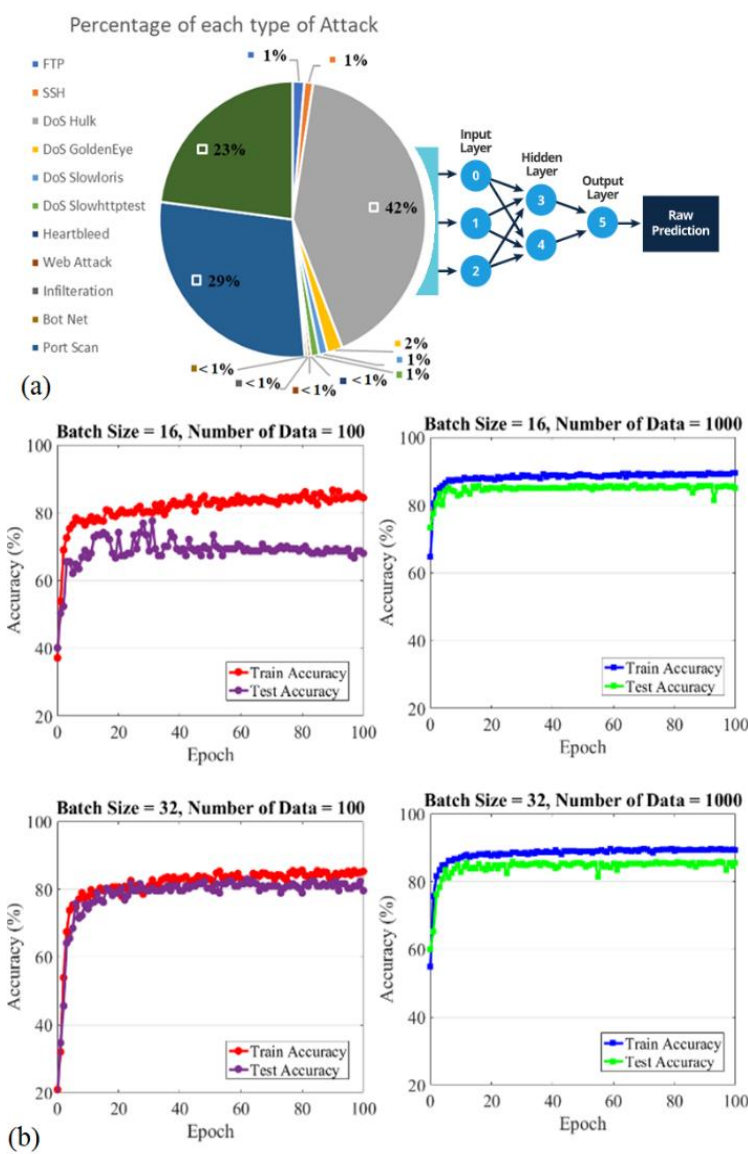

Figure 4: (a) percentage break down of each type of attack data included in the dataset; (b) Accuracy versus epoch for the learning rate of 0.1 and different values of batch size and the number of training data.

\section{Conclusion}

Machine learning is an ideal research field to promote innovation across a wide range of disciplinary domains. The author examines the educational strategies for engaging STEM students in ML research through developing supervised undergraduate research experiences (SURE) program and demonstrates how numerous interdisciplinary capstone projects can be developed in disciplinary domains, including Mechanical Engineering, Biology, Physics, and Cybersecurity. The paper explains four developed projects to enable the potential of ML for improving additive manufacturing, automating microscopy images analysis, identifying the quantum optical modes, and detecting IoT network intrusion. The author addresses the significant role of ML in the undergraduate STEM capstone projects besides the importance of improving and introducing comprehensive computing and data curricula in these majors. The paper provides the essential guidelines to effectively educate the next generation of STEM students who can efficiently solve big data problems in their disciplines and confront the new challenges in a computationally driven world.

\section{References}

[1] Langdon D., McKittrick G., Beede D., Khan B., and Doms M. (2011), "STEM: Good Jobs Now and for the Future. ESA Issue Brief\# 03-11," US Department of Commerce.

[2] Fayer S., Lacey A., and Watson A. (2017) "STEM occupations: Past, present, and future," Spotlight on Statistics, pp. 1-35.

[3] McKinsey G. (2011) "Big data: The next frontier for innovation, competition, and productivity," McKinsey Global Institute, pp. 1-6.

[4] Jindal R. (2014) "Louisiana Workforce Commission Employment forecasts by occupation and industry workforce investment council,"http://www.laworks.n et/Downloads/PR/WIC/WICPresentation20140610.pd f.

[5] Education C. (2011) "Future Jobs: A Look at National, State, and Congressional District Data," National Center for Women \& Informaton Technology.

[6] Code.org (2019) "Support K-12 Computer Science Education in Louisiana," Advocacy Coalition and CSTA State of Computer Science Education, https://code.org/advocacy/state-facts/LA.pdf.

[7] Charleston L., Adserias R. P., Lang N. M., and Jackson J. F. (2014) "Intersectionality and STEM: The role of race and gender in the academic pursuits of African American women in STEM," Journal of Progressive Policy \& Practice, 2(3), pp. 273-293.

[8] Judd B. C. and Graves C. A. (2012) "Cellular STEM: Promoting interest in science, technology, engineering, and math education using cellular messaging, cloud computing, and web-based social networks," 12th 
IEEE/ACM Intl Symp. on Cluster, Cloud and Grid Computing, pp. 799-804.

[9] Wang F., Kinzie M. B., McGuire P., and Pan E. (2010) "Applying technology to inquiry-based learning in early childhood education," Early Childhood Education Journal, 37(5), pp. 381-389.

[10] Davis D. (2014) "10 Years of Advanced Placement Exam Data Show Significant Gains in Access and Success; Areas for Improvement," The College Board Communications Office, 11.

[11] Lee I., Martin F., Denner J., Coulter B., Allan W., and Erickson J., and Werner, L. (2011) "Computational thinking for youth in practice," Acm Inroads, 2(1), pp. 32-37.

[12] B. Mueller (2012) "Additive manufacturing technologies-Rapid prototyping to direct digital manufacturing," Assembly Automation, Springer.

[13] Simonyan K. and Zisserman A. (2014) "Very deep convolutional networks for large-scale image recognition," arXiv preprint arXiv:1409.1556.

[14] Sadanandan S. K., Ranefall P., Le Guyader S., and Wählby C. (2017) "Automated training of deep convolutional neural networks for cell segmentation," Scientific reports, 7(1), pp. 1-7.

[15] Rostam H. M., Reynolds P. M., Alexander M. R., Gadegaard N., and Ghaemmaghami A. M. (2017) "Image based machine learning for identification of macrophage subsets," Scientific reports, 7(1), pp. 1-11.

[16] Ndagano B., Mphuthi N., Milione G., and Forbes A. (2017) "Comparing mode-crosstalk and modedependent loss of laterally displaced orbital angular momentum and Hermite-Gaussian modes for freespace optical communication," Optics letters, 42(20), pp. 4175-4178.

[17] Canedo J. and Skjellum A. (2016) "Using machine learning to secure IoT systems," 14th Annual Conf. on Privacy, Security and Trust (PST), pp. 219-222.

[18] Hasan M., Islam M. M., Zarif M. I. I., and Hashem M. (2019) "Attack and anomaly detection in IoT sensors in IoT sites using machine learning approaches," Internet of Things, 7, p. 100059,.

[19] Lawal M. A., Shaikh R. A., and Hassan S. R. (2020) "Security analysis of network anomalies mitigation schemes in IoT networks," IEEE Access, 8, pp. 4335543374.

[20] Panigrahi R. and Borah S. (2018) "A detailed analysis of CICIDS2017 dataset for designing Intrusion Detection Systems," International Journal of Engineering \& Technology, 7(3), pp. 479-482. 